\title{
M-estimates of location for the robust central tendency of fuzzy data
}

\author{
Beatriz Sinova, María Ángeles Gil and Stefan Van Aelst
}

\begin{abstract}
The Aumann-type mean has been shown to possess valuable properties as a measure of the location or central tendency of fuzzy data associated with a random experiment. However, concerning robustness its behaviour is not appropriate. The Aumann-type mean is highly affected by slight changes in the fuzzy data or when outliers arise in the sample. Robust estimators of location, on the other hand, avoid such adverse effects. For this purpose, this paper considers the M-estimation approach and discusses conditions under which this alternative yields valid fuzzy-valued M-estimators. The resulting M-estimators are applied to a real-life example. Finally, some simulation studies show empirically the suitability of the introduced estimators.
\end{abstract}

Index Terms - fuzzy number-valued data, M-estimators, random fuzzy numbers, robust location of fuzzy data.

\section{INTRODUCTION}

I $\mathrm{N}$ the current information age, new types of data emerge and we have to face the new challenging problems associated with the analysis of these data. Among these new types of data, fuzzy number-valued outcomes are more and more used in real-life applications to model data from intrinsically imprecise attributes.

When analyzing fuzzy data from a statistical viewpoint, the interest is often focused on the location (central tendency) of the random mechanism that generated the data. More concretely, fuzzy data can be assumed to be measurements of a random fuzzy number (fuzzy random variable in Puri and Ralescu's sense [1]) and the statistical analysis may concern the location of the distribution of this fuzzy number-valued random element.

This research has been partially supported by the Principality of Asturias/FEDER Grants GRUPIN14-101, Research Contract E-33-2015-0040746 (Sinova), the Spanish Ministry of Economy and Competitiveness Grant MTM2013-44212-P (Sinova) and a Grant of the Fund for Scientific ResearchFlanders (FWO-Vlaanderen) and an IAP research network grant nr. P7/06 of the Belgian government (Belgian Science Policy). Their support is gratefully acknowledged.

Authors are grateful to Colegio San Ignacio in Oviedo-Asturias (Spain), its Staff and its fourth grade students and parents for their support and understanding, which have allowed us to collect the data in the real-life example. Actually, we should say that these 9-10 years old students have quite properly caught the spirit and the mechanics behind fuzzy rating scalebased questionnaires.

Authors are also grateful to the reviewers and Associate Editor handling the original of this paper, as well as to their colleagues Professors Carlos Carleos and Pedro Terán, for their insightful comments and suggestions.

Sinova and Gil are with the Department of Statistics, O.R. and D.M. of the University of Oviedo, 33071 Asturias, Spain, e-mail: magil@uniovi.es, (see http://bellman.ciencias.uniovi.es/SMIRE), Van Aelst is with the Department of Mathematics, KU Leuven (University of Leuven), 3001 Leuven, Belgium, and Sinova and Van Aelst are with the Department of Applied Mathematics, Computer Science and Statistics, Ghent University, 9000 Gent, Belgium.

Manuscript received February 14, 2015; revised July 18, 2015; accepted September 8, 2015.
A well-known measure of the location of the distribution of a random fuzzy number is its Aumann-type mean (Puri and Ralescu [1]), which satisfies that

- it is well-defined under quite general conditions,

- it extends the mean value of a real-valued random variable,

- it preserves the main valuable properties of the realvalued mean (it is a 'linear' operator - i.e., it is equivariant under fuzzy arithmetic-based linear transformations-, it fulfils Fréchet's principle w.r.t. most of the $L^{2}$ metrics between fuzzy numbers, and it is coherent with the usual fuzzy arithmetic).

However, the Aumann-type mean also inherits from the mean of a real-valued random variable its high sensitivity to either the existence of outliers or small changes in the data. When looking for a more robust location measure, it seems convenient to follow successful approaches that were developed for other types of data. In this respect, this paper focuses on the well-known M-estimation approach.

To adapt the M-estimation approach to the case where the available data are fuzzy number-valued, two methodologies can be considered, namely:

- By using a suitable $L^{2}$ metric, fuzzy data can be represented as functional data resulting from (a convex cone within) certain Hilbert space-valued random elements (see, for instance, González-Rodríguez et al. [2] and Sinova et al. [3]). Consequently, we may adopt existing results and methods from Functional Data Analysis (more specifically, Kim and Scott's ideas and results [4], [5]) to the fuzzy case. This approach is valid whenever one can guarantee that the resulting measure cannot move out of the cone of the fuzzy data.

- Ad hoc fuzzy-valued M-estimators can be developed by using particular metrics based on specific representations of fuzzy numbers. For these representations there should exist sets of necessary and sufficient conditions which guarantee that the representation corresponds to a fuzzy number. Two ad hoc M-estimators have been introduced in Sinova et al. [6], [7] by using convenient $L^{1}$ metrics between fuzzy data. The corresponding fuzzy-valued Mestimators are quite easy to compute since their level sets can be expressed in terms of medians of realvalued random variables. It has also been shown that they preserve valuable properties of the median of a realvalued random variable, such as its robustness and strong consistency. 
This paper aims to approach the problem by using Mestimators which have been proposed in the area of Functional Data Analysis. Some recent proposals in the context of robust nonparametric density estimation connect ideas from traditional kernel density estimation with standard M-estimation. These proposals can be adapted to Hilbert space-valued random elements and they can be shown to yield valid estimators when restricted to random fuzzy numbers.

The relevant concepts about fuzzy number-valued data and random fuzzy numbers, i.e. one-dimensional random fuzzy sets are recalled in Section II. In Section III M-estimators of location for random fuzzy numbers are formally introduced. Necessary and sufficient conditions guaranteeing their existence, as well as their expression as a weighted mean of the fuzzy-valued sample data are provided. This feature is crucial for fuzzy data to guarantee that the corresponding Mestimators are fuzzy-valued under the considered conditions. Properties of the M-estimators are examined in detail. The weighted mean expression for M-estimators allows us to extend the iteratively re-weighted least squares algorithm used in classical M-estimation for the computation of sample location M-estimates for fuzzy data, as is illustrated by means of an example. Although the conditions for the existence of the Mestimators cover the extension of several important classical M-estimators, some recently proposed ad hoc M-estimators do not satisfy these conditions. We recall these proposals here for reasons of comparison. The use of fuzzy-valued M-estimators of location is illustrated by means of a real-life example.

Section IV presents the results of several simulation studies that have been carried out to empirically compare the robustness behavior of the location M-estimators introduced in this paper to the ad hoc proposals. Some concluding remarks are gathered in Section V.

\section{FUZZY DATA AND RANDOM FUZZY NUMBERS}

Fuzzy data appear in several domains. They are mainly used to describe imprecise human assessments such as ratings, opinions, judgments or perceptions in a natural and easy-tohandle way. In particular, when conducting quality ratings, satisfaction valuations and many other surveys, it can often not be expected that responses are expressed as fixed values in a precise scale, since they are essentially imprecise (see e.g. De la Rosa de Sáa et al. [8] for a recent detailed discussion about this point).

Fuzzy number-valued data can be interpreted as a 'levelwise' extension of interval-valued data, where the levels add a certain gradualness to the imprecision of interval-valued data. Formally, they are defined as follows:

Definition II.1. $\mathcal{F}_{c}^{*}(\mathbb{R})$ is the space of the (bounded) fuzzy numbers, that is, the mappings $\widetilde{U}: \mathbb{R} \rightarrow[0,1]$ such that their $\alpha$-levels

$$
\widetilde{U}_{\alpha}= \begin{cases}\{x \in \mathbb{R}: \widetilde{U}(x) \geq \alpha\} & \text { if } \alpha \in(0,1] \\ \operatorname{cl}\{x \in \mathbb{R}: \widetilde{U}(x)>0\} & \text { if } \alpha=0,\end{cases}
$$

are nonempty compact intervals. $\widetilde{U}(x)$ can be interpreted as the 'degree of compatibility' of $x$ with $\widetilde{U}$ ' (or 'degree of truth' of the assertion " $x$ is $\widetilde{U}$ ”).
To perform statistics with fuzzy data, one of the key tools is given by the arithmetic to operate with these data. Most of the theoretical and practical studies with fuzzy data consider the usual and natural approach corresponding to Zadeh's extension principle, which in this case extends level-wise the usual interval arithmetic.

Definition II.2. Let $\widetilde{U}, \widetilde{V} \in \mathcal{F}_{c}^{*}(\mathbb{R})$. The sum of $\widetilde{U}$ and $\widetilde{V}$ is defined as the fuzzy number $\widetilde{U}+\widetilde{V} \in \mathcal{F}_{c}^{*}(\mathbb{R})$ given for each $\alpha \in[0,1]$ by

$$
\begin{gathered}
(\widetilde{U}+\widetilde{V})_{\alpha}=\text { Minkowski sum of } \widetilde{U}_{\alpha} \text { and } \widetilde{V}_{\alpha} \\
=\left[\inf \widetilde{U}_{\alpha}+\inf \widetilde{V}_{\alpha}, \sup \widetilde{U}_{\alpha}+\sup \widetilde{V}_{\alpha}\right] .
\end{gathered}
$$

Let $\widetilde{U} \in \mathcal{F}_{c}^{*}(\mathbb{R})$ and $\gamma \in \mathbb{R}$. The product of $\widetilde{U}$ by the scalar $\gamma$ is defined as the fuzzy number $\gamma \cdot \widetilde{U} \in \mathcal{F}_{c}^{*}(\mathbb{R})$ given for each $\alpha \in[0,1]$ by

$(\gamma \cdot \widetilde{U})_{\alpha}=\gamma \cdot \widetilde{U}_{\alpha}= \begin{cases}{\left[\gamma \cdot \inf \widetilde{U}_{\alpha}, \gamma \cdot \sup \widetilde{U}_{\alpha}\right]} & \text { if } \gamma \geq 0 \\ {\left[\gamma \cdot \sup \widetilde{U}_{\alpha}, \gamma \cdot \inf \widetilde{U}_{\alpha}\right]} & \text { otherwise. }\end{cases}$

\section{A. Distances between fuzzy data}

It is well-known that when $\mathcal{F}_{c}^{*}(\mathbb{R})$ is endowed with this arithmetic it determines a semi-linear but not a linear space. As a consequence from this fact, there is no 'difference operation' between these values that is simultaneously well-defined and preserves the main properties of the difference between real values in connection with the sum.

Moreover, it should be pointed out that, although fuzzy numbers are formalized as $[0,1]$-valued functions, one cannot treat fuzzy data directly as if they were functional data in the way they are usually handled in Functional Data Analysis. This is due to to the fact that the functional arithmetic on $\mathcal{F}_{c}^{*}(\mathbb{R})$ can yield elements that lie outside this space and thus the meaning of fuzzy data is lost.

These concerns have been substantially overcome by developing statistical methods for imprecise data based on suitable distances between fuzzy numbers. On one hand, distances allow to 'translate' the equality of fuzzy numbers into the distance between these values being equal to 0 . On the other hand, appropriate distances also allow us to 'identify' fuzzy set-valued data with functional data through their so-called support function (see Puri and Ralescu [1]). Distances between fuzzy data have received a lot of attention in the literature. They have often been considered in connection with studies of similarity between fuzzy numbers, as well as for statistical purposes such as classification of fuzzy data or inferential statistics with fuzzy number-valued random elements.

In this paper, a parameterized and versatile family of distances has been chosen, although a few other distances will occasionally be mentioned. This family of distances has been introduced by Bertoluzza et al. [9] (see Casals et al. [10] for a recent review, and Trutschnig et al. [11] for its generalization to fuzzy vector-valued data). It is formalized as follows:

Definition II.3. Let $\theta \in(0,+\infty)$ and let $\varphi$ be an absolutely continuous probability measure on $\left([0,1], \mathcal{B}_{[0,1]}\right)$ with the mass function being positive on $(0,1)$. The mid/sprbased $L^{2}$ distance is defined as the mapping $D_{\theta}^{\varphi}: \mathcal{F}_{c}^{*}(\mathbb{R})$ 
$\times \mathcal{F}_{c}^{*}(\mathbb{R}) \rightarrow[0,+\infty)$ which associates with each pair $\widetilde{U}, \widetilde{V}$ of elements from $\mathcal{F}_{c}^{*}(\mathbb{R})$, the value

$$
\begin{gathered}
D_{\theta}^{\varphi}(\widetilde{U}, \widetilde{V})=\left[\int_{[0,1]}\left(\operatorname{mid} \widetilde{U}_{\alpha}-\operatorname{mid} \widetilde{V}_{\alpha}\right)^{2} d \varphi(\alpha)\right. \\
\left.+\theta \int_{[0,1]}\left(\operatorname{spr} \widetilde{U}_{\alpha}-\operatorname{spr} \widetilde{V}_{\alpha}\right)^{2} d \varphi(\alpha)\right]^{1 / 2},
\end{gathered}
$$

where $\operatorname{mid} \widetilde{U}_{\alpha}=\left(\inf \widetilde{U}_{\alpha}+\sup \widetilde{U}_{\alpha}\right) / 2$ and $\operatorname{spr} \widetilde{U}_{\alpha}$ $=\left(\sup \widetilde{U}_{\alpha}-\inf \widetilde{U}_{\alpha}\right) / 2$.

The mid/spr-based metric has been shown to be very suitable for the development of statistical methodology for experimental fuzzy set-valued data. For instance, GonzálezRodríguez et al. [2] provides a detailed explanation of an approach to ANOVA with fuzzy data based on the functional data identification. The recent reviews of Blanco-Fernández et al. [12], [13], [14], [15] and Gil et al. [16] summarize most of these statistical developments.

In these distances, differences in center and shape are taken into account through the mid and spr functions, respectively. The parameter $\theta$ and the measure $\varphi$ do not have a stochastic meaning. The parameter $\theta$ weighs the influence of the 'deviation in shape' between the fuzzy data with respect to the influence of their 'deviation in center'. Note that the choice $\theta=1$ corresponds to a generalization of the well-known distance $\rho_{2}$ by Diamond and Kloeden [17], which for any $\widetilde{U}, \widetilde{V}$ from $\mathcal{F}_{c}^{*}(\mathbb{R})$ is given by

$$
\begin{gathered}
D_{1}^{\varphi}(\widetilde{U}, \widetilde{V})=\rho_{\mathbf{2}}^{\varphi}(\widetilde{U}, \widetilde{V}) \\
=\left[\frac{1}{2} \int_{[0,1]}\left(\inf \widetilde{U}_{\alpha}-\inf \widetilde{V}_{\alpha}\right)^{2} d \varphi(\alpha)\right. \\
\left.+\frac{1}{2} \int_{[0,1]}\left(\sup \widetilde{U}_{\alpha}-\sup \widetilde{V}_{\alpha}\right)^{2} d \varphi(\alpha)\right]^{1 / 2} .
\end{gathered}
$$

Another interesting choice is associated with the value $\theta=1 / 3$, since (see Casals et al. [10]) it can be equivalently expressed as

$$
D_{1 / 3}^{\varphi}(\widetilde{U}, \widetilde{V})=\sqrt{\int_{[0,1]} \int_{[0,1]}\left(\widetilde{U}_{\alpha}^{[\eta]}-\widetilde{V}_{\alpha}^{[\eta]}\right)^{2} d \varphi(\alpha) d \ell(\eta)},
$$

with $\ell=$ Lebesgue measure on $\left([0,1], \mathcal{B}_{[0,1]}\right)$ and $\widetilde{U}_{\alpha}^{[\eta]}$ $=\eta \cdot \sup \widetilde{U}_{\alpha}+(1-\eta) \cdot \inf \widetilde{U}_{\alpha}$ for all $\left.\eta \in[0,1]\right)$.

The mid/spr-based distances are $L^{2}$ metrics that, through the support function (Puri and Ralescu's sense [1]), allow us to embed the space of fuzzy numbers into a convex cone of a Hilbert space by a Rådstrom-type isometry. More details can be found in Trutschnig et al. [11] and González-Rodríguez et al. [2] (see also Blanco-Fernández et al. [12] and Gil et al. [16] for recent reviews). In this way, despite the fact that fuzzy data should not be treated directly as functional data, they can be treated as functional data by considering the identification via the support function. As one can see later, this identification is crucial for the extension of M-estimators to fuzzy data.

\section{B. Random Fuzzy Numbers}

To formalize the statistical analysis of fuzzy data, an essential tool is the adaptation of a suitable model for the random mechanism generating fuzzy data. The notion of random fuzzy number in Puri and Ralescu's sense [18], [1] is a soundly established and well-supported model within the probabilistic setting. This notion is based on the ideas of Fréchet [19] and Féron [20]. In this model, fuzziness is assumed to affect the nature of values/data and randomness is assumed to affect their generation. Random fuzzy numbers can be defined as follows:

Definition II.4. Let $(\Omega, \mathcal{A}, P)$ be a probability space modeling a random experiment. A mapping $\mathcal{X}: \Omega \rightarrow \mathcal{F}_{c}^{*}(\mathbb{R})$ is said to be a random fuzzy number associated with the random experiment if, and only if, for each $\alpha \in[0,1]$ the intervalvalued mapping $\mathcal{X}_{\alpha}$ (where $\mathcal{X}_{\alpha}(\omega)=(\mathcal{X}(\omega))_{\alpha}$ for all $\omega \in \Omega$ ) is a random compact interval or equivalently, the real-valued functions $\inf \mathcal{X}_{\alpha}$ and $\sup \mathcal{X}_{\alpha}$ are random variables.

It should be highlighted that a random fuzzy number is a Borel-measurable mapping with respect to the Borel $\sigma$ field associated with several metrics like, for instance, the $\mathrm{mid} / \mathrm{spr}$-based distance (see, for instance, González-Rodríguez et al. [2] and Gil et al. [16]). As a consequence from the Borelmeasurability, crucial concepts such as the induced distribution of a random fuzzy number or the stochastic independence of random fuzzy numbers can be immediately stated.

The best known measure of location of a random fuzzy number is the Aumann-type mean, which is given by

Definition II.5. Let $\mathcal{X}$ be a random fuzzy number and assume that the expected values of the random variables inf $\mathcal{X}_{0}$ and sup $\mathcal{X}_{0}$ are finite. The Aumann-type mean of $\mathcal{X}$ is the fuzzy number $\widetilde{E}(\mathcal{X}) \in \mathcal{F}_{c}^{*}(\mathbb{R})$ such that for each $\alpha \in[0,1]$

$$
(\widetilde{E}(\mathcal{X}))_{\alpha}=\left[E\left(\inf \mathcal{X}_{\alpha}\right), E\left(\sup \mathcal{X}_{\alpha}\right)\right] .
$$

Note that this notion, introduced by Puri and Ralescu [1], is coherent with the usual fuzzy arithmetic. In particular, if the possible values of a random fuzzy number $\mathcal{X}$ are given by $\left\{\widetilde{x}_{1}, \ldots, \widetilde{x}_{m}\right\}$ and $P\left(\mathcal{X}=\widetilde{x}_{i}\right)=P\left(\left\{\omega \in \Omega: \mathcal{X}(\omega)=\widetilde{x}_{i}\right\}\right)$, then it holds that

$$
\widetilde{E}(\mathcal{X})=P\left(\mathcal{X}=\widetilde{x}_{1}\right) \cdot \widetilde{x}_{1}+\ldots+P\left(\mathcal{X}=\widetilde{x}_{m}\right) \cdot \widetilde{x}_{m} .
$$

That is,

$$
\begin{gathered}
(\widetilde{E}(\mathcal{X}))_{\alpha} \\
=\left[\sum_{i=1}^{m} \inf \left(\widetilde{x}_{i}\right)_{\alpha} \cdot P\left(\mathcal{X}=\widetilde{x}_{i}\right), \sum_{i=1}^{m} \sup \left(\widetilde{x}_{i}\right)_{\alpha} \cdot P\left(\mathcal{X}=\widetilde{x}_{i}\right)\right] .
\end{gathered}
$$

The Aumann-type mean of a random fuzzy number is supported by Strong Laws of Large Numbers (see e.g. Colubi et al. [21]) and also by the Fréchet approach [19], since it is the fuzzy number minimizing the 'mean square error' of $\mathcal{X}$ about a fuzzy number. That is,

$$
\widetilde{E}(\mathcal{X})=\arg \min _{\widetilde{U} \in \mathcal{F}_{c}^{*}(\mathbb{R})} E\left[\left(D_{\theta}^{\varphi}(\mathcal{X}, \widetilde{U})\right)^{2}\right]
$$

for every $\theta$ and $\varphi$ for which the expectation exists. 


\section{LOCATION M-ESTIMATES FROM FUZZY DATA}

M-estimation is a well-established approach that yields robust estimators. M-estimators were first introduced by $\mathrm{Hu}$ ber [22] to estimate the location of real-valued data with the aim of limiting the influence of outliers on methods like least squares and maximum likelihood. For this purpose, the key idea consists of replacing the square of the 'errors' with a (usually less rapidly increasing) loss function applied to the errors of the data. In this respect, M-estimators were presented as intermediaries between the sample mean and median.

In this section, the notion of M-estimators is extended to the case of fuzzy number-valued data. Sufficient conditions on the loss function to guarantee that the M-estimation process leads to well-defined (i.e., fuzzy-valued) estimates are established. Following Huber's ideas [22], [23], a location M-estimate in connection with a random fuzzy number is formalized as follows:

Definition III.1. Let $(\Omega, \mathcal{A}, P)$ be a probability space and $\mathcal{X}: \Omega \rightarrow \mathcal{F}_{c}^{*}(\mathbb{R})$ an associated random fuzzy number. Moreover, let $\rho$ be an arbitrary continuous real-valued function (which will be referred to hereinafter as the loss function), and $\left(\mathcal{X}_{1}, \ldots, \mathcal{X}_{n}\right)$ a simple random sample from $\mathcal{X}$. Then, the sample fuzzy M-estimator of location is the fuzzy numbervalued statistic $\widetilde{g}^{M}\left[\left(\widehat{\mathcal{X}_{1}, \ldots}, \mathcal{X}_{n}\right)\right]$, given by

$$
\widetilde{g}^{M}\left[\left(\widehat{\mathcal{X}_{1}, \ldots}, \mathcal{X}_{n}\right)\right]=\arg \min _{\widetilde{g} \in \mathcal{F}_{c}^{*}(\mathbb{R})} \frac{1}{n} \sum_{i=1}^{n} \rho\left(D_{\theta}^{\varphi}\left(\mathcal{X}_{i}, \widetilde{g}\right)\right),
$$

if it exists.

The $\rho$-function in Definition III.1 generalizes the squared loss function in the least squares approach. Actually, it is usually assumed to vanish at 0 and to be non-decreasing.

If the whole class of fuzzy numbers $\mathcal{F}_{c}(\mathbb{R})$ (including unbounded ones, with non-compact 0-level) is considered, it is possible to prove that the M-estimator of location is Borel measurable, and therefore, a statistic.

Proposition III.1. Let $(\Omega, \mathcal{A}, P)$ be a Polish probability space and $\mathcal{X}: \Omega \rightarrow \mathcal{F}_{c}(\mathbb{R})$ an associated random fuzzy number. Moreover, let $\left(\mathcal{X}_{1}, \ldots, \mathcal{X}_{n}\right)$ be a simple random sample from $\mathcal{X}$ and $\rho$ a continuous loss function. Then, the M-estimator of location is well-defined.

It is very common in practice to fix a bounded referential in $\mathbb{R}$, as is the case for the fuzzy rating scale by Hesketh et al. [37]. When adopting this situation and working on $\mathcal{F}_{c}([a, b])$, for certain $a, b \in \mathbb{R}$ such that $a<b$, instead of $\mathcal{F}_{c}(\mathbb{R})$, Proposition III.1 also holds.

To show that Definition III.1 yields valid estimators for the location of fuzzy random variables, we have to show that the estimators are indeed fuzzy-valued statistics. This is an immediate corollary of the following representer theorem.

\section{A. Representer theorem for M-estimates with fuzzy data}

Kim and Scott [4], [5] recently proposed a robust nonparametric density estimator by combining a traditional kernel density estimator with ideas from standard M-estimation. An interesting contribution of Kim and Scott's work lies in their analysis of the conditions to ensure the existence of sample $\mathrm{M}$-estimates of location. These conditions allow us to express the M-estimates as weighted linear combinations of the sample elements. This feature will be very convenient to assure that the M-estimates are indeed fuzzy number-valued statistics.

The novel representer theorem that will be stated in Theorem III-A.1 is an adaptation of Kim and Scott's work [4], [5] to the fuzzy-valued case by means of the isometrical embedding mentioned before. It expresses the sample location M-estimate for fuzzy number-valued data as a convex linear combination of the sample components, $\widetilde{x}_{1}, \ldots, \widetilde{x}_{n}$.

Theorem III-A.1. Consider $\mathcal{F}_{c}^{*}(\mathbb{R})$ endowed with the metric $D_{\theta}^{\varphi}$. Let $\widetilde{\mathbf{x}}_{\mathbf{n}}=\left(\widetilde{x}_{1}, \ldots, \widetilde{x}_{n}\right)$ be a sample of independent observations from a random fuzzy number $\mathcal{X}: \Omega \rightarrow \mathcal{F}_{c}^{*}(\mathbb{R})$ on a probability space $(\Omega, \mathcal{A}, P)$. Moreover, let $\rho$ be a continuous loss function which satisfies the assumptions

- $\rho$ is non-decreasing, $\rho(0)=0$ and $\lim _{x \rightarrow 0} \rho(x) / x=0$,

- Let $\phi(x)=\rho^{\prime}(x) / x$ and $\phi(0) \equiv \lim _{x \rightarrow 0} \phi(x)$. Then $\phi(0)$ exists and it is finite.

Then, the sample M-estimate of location exists and it can be expressed as

$$
\widehat{\widetilde{g}^{M\left[\widetilde{\mathbf{x}}_{\mathbf{n}}\right]}}=\sum_{i=1}^{n} w_{i} \cdot \widetilde{x}_{i}
$$

with $w_{i} \geq 0$ and $\sum_{i=1}^{n} w_{i}=1$. Furthermore,

$$
w_{i} \propto \phi\left(D_{\theta}^{\varphi}\left(\widetilde{x}_{i}, \widehat{\widetilde{g}^{M}\left[\widetilde{\mathbf{x}}_{\mathbf{n}}\right]}\right)\right) .
$$

Many loss functions $\rho$ that are used to compute Mestimators satisfy the above-mentioned conditions. One of the most well-known loss functions which fulfills the required conditions is the Huber loss function [23], which is given by

$$
\rho_{a}(x)=\left\{\begin{array}{ll}
x^{2} / 2 & \text { if }|x| \leq a \\
a(|x|-a / 2) & \text { otherwise, }
\end{array},\right.
$$

with $a>0$ a tuning parameter. Note that the Huber loss function is convex, but not strictly convex. Huber's loss function yields a hybrid approach between squared and absolute error losses. Indeed, it corresponds to a parabola in the vicinity of 0 and increases linearly from the given level $a$ onwards, so that one can put appropriate emphasis on large and small errors. Some discussions on the choice of the tuning parameter $a$ can be found in e.g. Wang et al. [25] and Debruyne et al. [26].

Another well-known loss function is the Hampel loss function [27], which corresponds to

$$
\rho_{a, b, c}(x)= \begin{cases}x^{2} / 2 & \text { if } 0 \leq|x|<a \\ a(|x|-a / 2) & \text { if } a \leq|x|<b \\ \frac{a(|x|-c)^{2}}{2(b-c)}+\frac{a(b+c-a)}{2} & \text { if } b \leq|x|<c \\ \frac{a(b+c-a)}{2} & \text { if } c \leq|x|,\end{cases}
$$

where the nonnegative parameters $a<b<c$ allow us to control the degree of suppression of large errors. The smaller their values, the higher this degree. 
The performance of M-estimators thus depends on the choice on the tuning parameter(s) that appear in both loss functions. To fix these tuning parameter(s), one can follow the ideas in Kim and Scott [5], that is,

1) Choose as starting solution an easily computable robust centrality measure (to ensure the robustness of the Mestimator unless we can assure that the M-estimator is unique);

2) Compute the distances between the fuzzy observations and this initial solution;

3) Set $a$ equal to the median of these real-valued distances for both loss functions. For Hampel's loss function, set $b$ and $c$ equal to the $75^{t h}$ and the $85^{t h}$ percentiles of the obtained distances, respectively.

An example of the Huber loss function for $a=2.36$ (used in Example III.1) is shown in Figure 1. Figure 1 displays the loss function $\rho_{2.36}$, its derivative $\rho_{2.36}^{\prime}$ and $\phi_{2.36}$ (corresponding to the quotient of $\rho_{2.36}^{\prime}$ and the identity function).

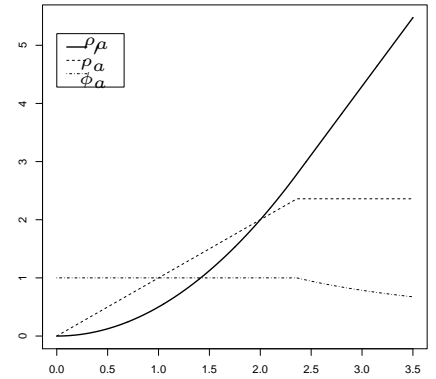

Fig. 1. Huber loss function $\rho_{2.36}$, its derivative $\rho_{2.36}^{\prime}$ and $\phi_{2.36}$

To illustrate the Hampel loss function for the values of the tuning parameters in Example III.1 below, Figure 2 displays the loss function $\rho_{2.36,3.18,3.51}$, its derivative $\rho_{2.36,3.18,3.51}^{\prime}$ and $\phi_{2.36,3.18,3.51 \text {. }}$
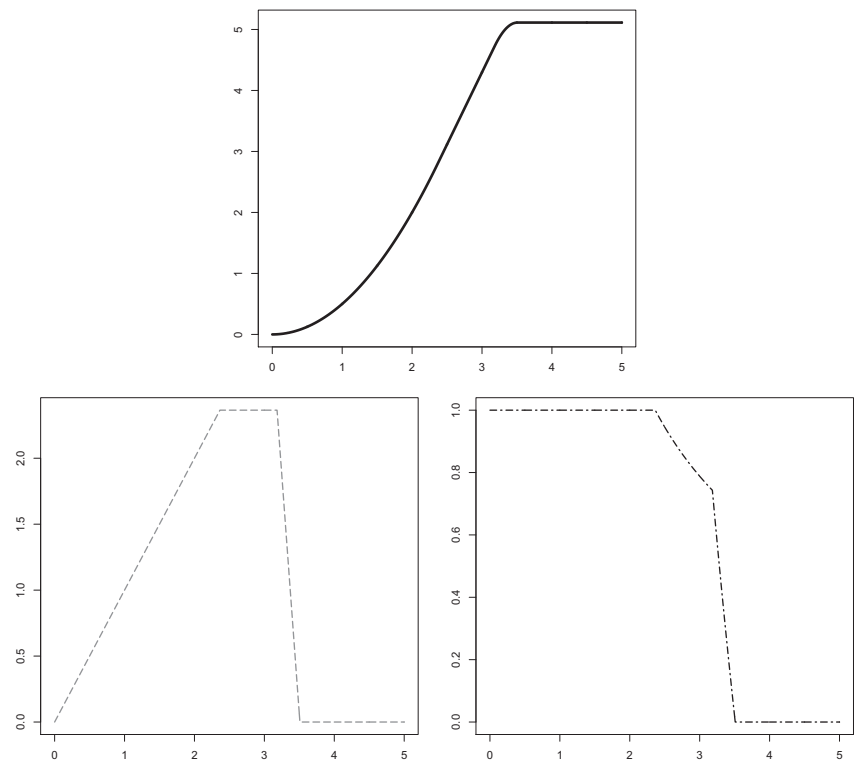

Fig. 2. Hampel loss function $\rho_{a, b, c}$ (top), its derivative $\rho_{a, b, c}^{\prime}$ (bottom left) and $\phi_{a, b, c}$ (bottom right) with $a=2.36, b=3.18$ and $c=3.51$
The following (artificial) example explains the algorithm to compute the M-estimates corresponding to the Huber loss function $\rho_{2.36}$ and to the Hampel loss function $\rho_{2.36,3.18,3.51}$ based on the $L^{2}$ metric $D_{1 / 3}^{\ell}$.

Example III.1. Consider a sample $\widetilde{\mathbf{x}}_{3}=\left(\widetilde{x}_{1}, \widetilde{x}_{2}, \widetilde{x}_{3}\right)$ with three trapezoidal/triangular fuzzy values given by $\widetilde{x}_{1}$ $=\operatorname{Tra}(0,2,3,4), \widetilde{x}_{2}=\operatorname{Tri}(1,1.5,2)$ and $\widetilde{x}_{3}=\operatorname{Tri}(3,4,5)$ (displayed in grey in Figure 3).

The parameter values are fixed to $a=2.36, b=3.18$ and $c=3.51$. For the Huber loss function $\rho_{2.36}$, the corresponding M-estimate of location can then be determined by means of the following algorithm:

Step 1. Take as initial estimate $\widetilde{g}_{(1)}^{M}(k=1)$ a 'central' value. In this case, we have chosen the 1-norm median (see Subsection III-C) as initial robust location measure. Fix a tolerance $\varepsilon=10^{-7}$.

Step 2. Update the weights

$$
w_{i}^{(k)}=\frac{\phi\left(D_{1 / 3}^{\ell}\left(\widetilde{x}_{i}, \widetilde{g}_{(k)}^{M}\right)\right)}{\sum_{j=1}^{n} \phi\left(D_{1 / 3}^{\ell}\left(\widetilde{x}_{j}, \widetilde{g}_{(k)}^{M}\right)\right)}
$$

and the estimate

$$
\widetilde{g}_{(k+1)}^{M}=\sum_{i=1}^{n} w_{i}^{(k)} \cdot \widetilde{x}_{i} .
$$

Step 3. Repeat Step 2 until

$$
\frac{\left|J\left(\widetilde{g}_{(k+1)}^{M}\right)-J\left(\widetilde{g}_{(k)}^{M}\right)\right|}{J\left(\widetilde{g}_{(k)}^{M}\right)}<\varepsilon .
$$

The final estimate is given by the trapezoidal fuzzy number $\operatorname{Tra}(1.33,2.50,2.83,3.66)$, which has been displayed in black (straight line) in Figure 3.

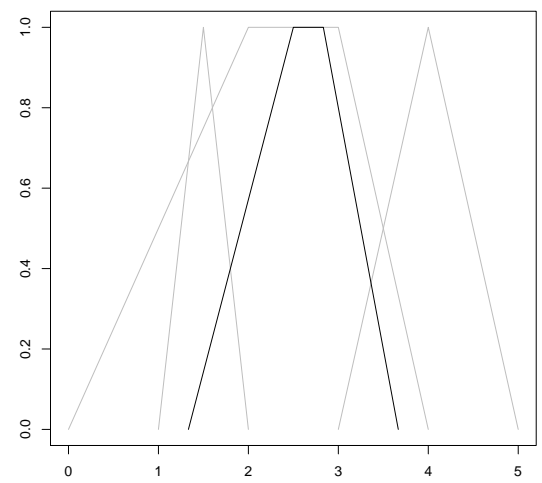

Fig. 3. A Huber (black line) M-estimate of location of the sample of the grey fuzzy data (using $D_{1 / 3}^{\ell}$ )

Analogously, the corresponding M-estimate of location for the Hampel loss function $\rho_{2.36,3.18,3.51}$ can be calculated, which in this example is almost indistinguishable from the solution for the Huber loss function.

\section{B. Properties of M-estimates satisfying the representer theorem}

In this subsection, we state several interesting properties of the fuzzy-valued M-estimates when the conditions of the 
representer theorem are satisfied. These properties have not been studied by Kim and Scott in the context of density estimation yet. Proofs can be found as the supplementary material.

In particular, the M-estimates are translation equivariant and under additional assumptions also scale (and, hence, symmetry) equivariant.

Proposition III-B.1. (Translation equivariance) Consider $\mathcal{F}_{c}^{*}(\mathbb{R})$ endowed with the metric $D_{\theta}^{\varphi}$. Let $\widetilde{\mathbf{x}}_{\mathbf{n}}=\left(\widetilde{x}_{1}, \ldots, \widetilde{x}_{n}\right)$ be a sample of independent observations from a random fuzzy number $\mathcal{X}: \Omega \rightarrow \mathcal{F}_{c}^{*}(\mathbb{R})$ on a probability space $(\Omega, \mathcal{A}, P)$. Moreover, let $\rho$ be an arbitrary continuous loss function fulfilling the assumptions in Theorem III-A.1, such that the corresponding sample M-estimate of location is unique.

Let $\widetilde{U} \in \mathcal{F}_{c}^{*}\left(\underset{\mathbb{R}}{)}\right.$. If $\left.\widetilde{g}^{M} \widehat{\left[\widetilde{\mathbf{x}}_{\mathbf{n}}+\right.} \widetilde{U}\right]$ is the location M-estimate based on $\widetilde{\mathbf{x}}_{\mathbf{n}}+\widetilde{U}$, then it holds that

$$
\left.\widetilde{g^{M}} \widehat{\left[\widetilde{\mathbf{x}}_{\mathbf{n}}+\right.} \widetilde{U}\right]=\widetilde{\widetilde{g}^{M\left[\widetilde{\mathbf{x}}_{\mathbf{n}}\right]}}+\widetilde{U}
$$

where $\widehat{\widetilde{g}^{M}\left[\widetilde{\mathbf{x}}_{\mathbf{n}}\right]}$ is the location M-estimate based on $\widetilde{\mathbf{x}}_{\mathbf{n}}$, which satisfies

i) $\widehat{\left.\widetilde{g}^{M\left[\widetilde{\mathbf{x}}_{\mathbf{n}}\right.}\right]}=\sum_{i=1}^{n} w_{i} \cdot \widetilde{x}_{i}$,

ii) $w_{i} \propto \phi\left(D_{\theta}^{\varphi}\left(\widetilde{x}_{i}, \widehat{g^{M}\left[\widetilde{\mathbf{x}}_{\mathbf{n}}\right]}\right)\right)$,

iii) $\sum_{i=1}^{n} w_{i}=1$.

Proposition III-B.2. (Scale equivariance) Consider $\mathcal{F}_{c}^{*}(\mathbb{R})$ endowed with the metric $D_{\theta}^{\varphi}$. Let $\widetilde{\mathbf{x}}_{\mathbf{n}}=\left(\widetilde{x}_{1}, \ldots, \widetilde{x}_{n}\right)$ be a sample of independent observations from a random fuzzy number $\mathcal{X}: \Omega \rightarrow \mathcal{F}_{c}^{*}(\mathbb{R})$ on a probability space $(\Omega, \mathcal{A}, P)$. Moreover, let $\rho$ be an arbitrary continuous loss function fulfilling the assumptions in Theorem III-A.1, such that the corresponding sample M-estimate of location is unique.

If $\rho$ satisfies the condition $\phi(k \cdot x) \propto \phi(x)$ for every $k$ $\in(0, \infty)$ (i.e., the coefficient of proportionality is independent of $x$ ), then for all $c \in \mathbb{R}$ it holds that

$$
\widetilde{g}^{M\left[c \cdot \widetilde{\mathbf{x}}_{\mathbf{n}}\right]}=c \cdot \widehat{\left.\widetilde{g}^{M\left[\widetilde{\mathbf{x}}_{\mathbf{n}}\right.}\right]},
$$

where $\widetilde{g}^{M\left[c \cdot \widetilde{\mathbf{x}}_{\mathbf{n}}\right]}$ is the location M-estimate based on $c \cdot \widetilde{\mathbf{x}}_{\mathbf{n}}$, and $\widetilde{g}^{M\left[\widetilde{\mathbf{x}}_{\mathbf{n}}\right]}$ is the location M-estimate based on $\widetilde{\mathbf{x}}_{\mathbf{n}}$, which satisfies

i) $\widehat{\left.\widetilde{g}^{M\left[\widetilde{\mathbf{x}}_{\mathbf{n}}\right.}\right]}=\sum_{i=1}^{n} w_{i} \cdot \widetilde{x}_{i}$,

ii) $w_{i} \propto \phi\left(D_{\theta}^{\varphi}\left(\widetilde{x}_{i}, \widehat{g^{M}\left[\widetilde{\mathbf{x}}_{\mathbf{n}}\right]}\right)\right)$,

iii) $\sum_{i=1}^{n} w_{i}=1$.

For instance, if $\phi$ is a power function, then the corresponding M-estimate is scale equivariant.

Note that the extra condition on the loss function in Theorem III-B.2 is a strong requirement. Most loss functions for M-estimates of location do not satisfy this property. Therefore, similarly as in the real setting (see e.g. Maronna et al. [28]) the resulting M-estimate, especially its robustness, may depend heavily on the considered measurement units. To avoid this drawback, we determine the values of the tuning parameters in the considered loss functions based on the distribution of the distances for the particular dataset, as already explained after Theorem III-A.1.
As an immediate consequence from the representer theorem for fuzzy data, one can trivially prove that the M-estimates of $L R$ fuzzy numbers sharing $L$ and $R$ functions (see Dubois and Prade [29]) also fall in the $L R$ family. Recall that $\widetilde{U}$ is said to be an $L R$ fuzzy number $(b, c, b-a, d-c)_{L R}$ if

$$
\widetilde{U}(x)= \begin{cases}L\left(\frac{b-x}{b-a}\right) & \text { if } x \in[a, b) \\ 1 & \text { if } x \in[b, c] \\ R\left(\frac{x-c}{d-c}\right) & \text { if } x \in(c, d] \\ 0 & \text { otherwise }\end{cases}
$$

where $a, b, c, d \in \mathbb{R}, a \leq b \leq c \leq d$, and $L, R:[0,1]$ $\rightarrow \quad[0,1]$ are continuous non-increasing functions such that $L(x)=R(y)=1$ iff $x=y=0$ and $L(x)=R(y)=0$ iff $x=y=1)$.

Proposition III-B.3. Assume the conditions in Theorem III-A.1 are fulfilled. Assume also that $\widetilde{\mathbf{x}}_{\mathbf{n}}=\left(\widetilde{x}_{1}, \ldots, \widetilde{x}_{n}\right)$ is a sample of independent observations such that all $\widetilde{x}_{i}$ 's are $L R$ fuzzy numbers sharing the $L$ and $R$ functions. Then, the sample M-estimate of location is an LR fuzzy number with the same $L$ and $R$ functions. In particular, if all $\widetilde{x}_{i}$ 's are trapezoidal fuzzy numbers, then the sample M-estimate of location is trapezoidal as well.

Another interesting property when examining the adequacy of the location M-estimates based on fuzzy data as central tendency measures concerns their behavior in case of symmetrically distributed samples. In the real-valued case, a well-known result is that the median of a symmetric random variable coincides with the point of symmetry whenever it is unique. In the fuzzy-valued case, a similar result can be obtained for location M-estimates under certain assumptions.

Let $\widetilde{\mathbf{x}}_{\mathbf{n}}=\left(\widetilde{x}_{1}, \ldots, \widetilde{x}_{n}\right)$ be a sample of independent observations from a random fuzzy number $\mathcal{X}: \Omega \rightarrow \mathcal{F}_{c}^{*}(\mathbb{R})$ on a probability space $(\Omega, \mathcal{A}, P)$. Then, the sample $\widetilde{\mathbf{x}}_{\mathbf{n}}$ is said to be symmetric about $c \in \mathbb{R}$ (see Sinova et al. [30]) if and only if $\widetilde{\mathbf{x}}_{\mathbf{n}}-c$ and $c-\widetilde{\mathbf{x}}_{\mathbf{n}}$ (or, equivalently, $2 c-\widetilde{\mathbf{x}}_{\mathbf{n}}$ and $\widetilde{\mathbf{x}}_{\mathbf{n}}$ ) include exactly the same fuzzy data. We then have the following result:

Proposition III-B.4. Consider $\mathcal{F}_{c}^{*}(\mathbb{R})$ endowed with the metric $D_{\theta}^{\varphi}$. Let $\widetilde{\mathbf{x}}_{\mathbf{n}}=\left(\widetilde{x}_{1}, \ldots, \widetilde{x}_{n}\right)$ be a sample of independent observations from a random fuzzy number $\mathcal{X}: \Omega \rightarrow \mathcal{F}_{c}^{*}(\mathbb{R})$ on a probability space $(\Omega, \mathcal{A}, P)$. Moreover, let $\rho$ be an arbitrary continuous loss function fulfilling the assumptions in Theorem III-A.1, such that the associate M-estimate is unique. If the sample $\widetilde{\mathbf{x}}_{\mathbf{n}}$ is symmetric about $c \in \mathbb{R}$, then the corresponding location M-estimate $\widetilde{g}^{M\left[c \cdot \widetilde{\mathbf{x}}_{\mathbf{n}}\right]}$ is a symmetric fuzzy number about $c$.

In addition to these properties, it is also of importance to investigate the consistency of sample M-estimators as well as their robustness.

Strong consistency concerns the almost sure convergence of sample M-estimators to their population analogue, given by

$$
\tilde{g}^{M}(\mathcal{X})=\arg \min _{\widetilde{U} \in \mathcal{F}_{c}^{*}(\mathbb{R})} E\left[\rho\left(D_{\theta}^{\varphi}(\mathcal{X}, \widetilde{U})\right)\right] .
$$


In the literature, consistency has been formally guaranteed under certain conditions (see e.g. Vandermeulen and Scott [31]). However, those results do not include most of the commonly used loss functions. In order to cover, among others, the well-known Huber and Hampel loss functions, the following theorem is now stated under the assumption that the referential fuzzy numbers are defined on is bounded referential, as happens when using the fuzzy rating scale.

Theorem III-B.1. Consider the metric space $\left(\mathcal{F}_{c}([a, b]), D_{\theta}^{\lambda}\right)$, with $a, b \in \mathbb{R}, a<b$ and $\theta>0$. Let $\mathcal{X}: \Omega \rightarrow \mathcal{F}_{c}^{*}(\mathbb{R})$ be a random fuzzy number associated with a probability space $(\Omega, \mathcal{A}, P)$. Under any of the following assumptions:

- $\rho$ is non-decreasing, subadditive and unbounded,

- $\rho$ is the Huber loss function (for any tuning parameter $a>0$ ),

- $\rho$ is the Hampel loss function (for any tuning parameters $c>b>a>0)$,

and whenever $\tilde{g}^{M}(\mathcal{X})$ exists and is unique, the sample $M$ estimator of location is a strongly consistent estimator of $\tilde{g}^{M}(\mathcal{X})$, i.e.,

$$
\lim _{n \rightarrow \infty} D_{\theta}^{\varphi}\left(\widetilde{g}^{M}\left[\left(\widehat{\mathcal{X}_{1}, \ldots}, \mathcal{X}_{n}\right)\right], \tilde{g}^{M}(\mathcal{X})\right)=0 \quad \text { a.s. }[P] .
$$

We now investigate the robustness of location M-estimators for fuzzy data by means of the finite sample breakdown point, for short fsbp (Hampel [32], Donoho and Huber [33]). The fsbp is a measure of robustness that can be adapted to estimators taking values in general metric spaces (Cuevas et al. [34]). It represents the smallest fraction of observations in a sample that needs to be perturbed to make the (suitably chosen) distance between the M-estimates in the original and contaminated samples arbitrarily large.

The first result shows the importance of the translational equivariance of M-estimators as stated in Proposition III-B.1.

Theorem III-B.2. Consider the metric space $\left(\mathcal{F}_{c}^{*}(\mathbb{R}), D_{\theta}^{\varphi}\right)$ and $\theta$ any nonnegative value. Let $\mathcal{X}: \Omega \rightarrow \mathcal{F}_{c}^{*}(\mathbb{R})$ be a random fuzzy number associated with a probability space $(\Omega, \mathcal{A}, P)$. Moreover, let $\rho$ be an arbitrary continuous loss function fulfilling the assumptions in Theorem III-A.1, such that the corresponding sample M-estimate of location is unique. Then, the finite sample breakdown point of the corresponding location M-estimator is at most $\frac{1}{n}\left\lfloor\frac{n+1}{2}\right\rfloor$, where $\lfloor\cdot\rfloor$ denotes the floor function.

Moreover, it is possible to prove that this upper bound for the finite sample breakdown point is attained under an additional sufficient condition on the loss function.

Theorem III-B.3. Consider the metric space $\left(\mathcal{F}_{c}^{*}(\mathbb{R}), D_{\theta}^{\varphi}\right)$ and $\theta$ any nonnegative value. Let $\mathcal{X}: \Omega \rightarrow \mathcal{F}_{c}^{*}(\mathbb{R})$ be a random fuzzy number associated with a probability space $(\Omega, \mathcal{A}, P)$. Moreover, let $\rho$ be a continuous loss function fulfilling the assumptions in Theorem III-A.1, such that the corresponding sample M-estimate of location is unique. Under any of these assumptions

- $\rho$ has linear upper and lower bounds;

- $\rho_{a, b, c}$ is a Hampel's loss function whose tuning parameters satisfy

$$
\rho_{a, b, c}\left(\max _{1 \leq i, j \leq n} D_{\theta}^{\varphi}\left(\widetilde{x}_{i}, \widetilde{x}_{j}\right)\right)<\frac{n-2\left\lfloor\frac{n-1}{2}\right\rfloor}{n-\left\lfloor\frac{n-1}{2}\right\rfloor-1} \cdot \rho_{a, b, c}(c) ;
$$

the finite sample breakdown point of the corresponding location M-estimator is exactly $\frac{1}{n}\left\lfloor\frac{n+1}{2}\right\rfloor$.

Notice that Theorem III-B.3 includes Huber's loss function, which is linear for large values of its argument and thus can be upper and lower bounded by straight lines. This result indicates that the fsbp of both Huber and Hampel M-estimates (the latter for sufficient large choices of the tuning parameters) is equal to $\frac{1}{n} \cdot\left\lfloor\frac{n+1}{2}\right\rfloor$. In contrast, the fsbp of the sample Aumann-type mean has been shown to be equal to $\frac{1}{n}$ (see e.g. Sinova et al. [6], [7]). M-estimates thus yield a huge gain in robustness in comparison to the commonly used Aumann-type mean.

\section{Related ad hoc estimators}

The representer theorem guarantees the existence of sample M-estimates of location as well as their expression as random weighted means of the sample elements for loss functions that satisfy the needed conditions. While several common choices of the loss function $\rho$ satisfy these conditions, there are also some interesting choices which do not satisfy these conditions. In particular, for the choice $\rho(x)=|x|$, which yields the median in the real-valued case, one cannot apply the results in Section III-A. However, Sinova et al. [6], [7] proposed median-type M-estimates for random fuzzy numbers. These M-estimates cannot be expressed as weighted means of the sample elements, but level-wise they can be expressed in terms of the classical medians of real-valued random variables characterizing the fuzzy number. This makes computation of the median-type M-estimators rather easy to accomplish in practice. In particular, Sinova et al. [6] introduced the 1-norm median as follows.

Definition III.2. Let $\widetilde{\mathbf{x}}_{\mathbf{n}}=\left(\widetilde{x}_{1}, \ldots, \widetilde{x}_{n}\right)$ be a sample of independent observations from a random fuzzy number $\mathcal{X}: \Omega$ $\rightarrow \mathcal{F}_{c}^{*}(\mathbb{R})$ on a probability space $(\Omega, \mathcal{A}, P)$. The sample $\mathbf{1}$ norm median of $\mathcal{X}$ is defined as the fuzzy number $\widetilde{\mathrm{Me}}\left[\widetilde{\mathbf{x}}_{\mathbf{n}}\right]$ $\in \mathcal{F}_{c}^{*}(\mathbb{R})$ such that for each $\alpha \in[0,1]$

$$
\begin{gathered}
\left(\widehat{\operatorname{Me}\left[\widetilde{\mathbf{x}}_{\mathbf{n}}\right]}\right)_{\alpha}=\left[\operatorname{Me}\left\{\inf \left(\widetilde{x}_{1}\right)_{\alpha}, \ldots, \inf \left(\widetilde{x}_{n}\right)_{\alpha}\right\},\right. \\
\left.\operatorname{Me}\left\{\sup \left(\widetilde{x}_{1}\right)_{\alpha}, \ldots, \sup \left(\widetilde{x}_{n}\right)_{\alpha}\right\}\right]
\end{gathered}
$$

(where in case the real-valued median Me is non-unique the mid-point convention is considered).

The 1-norm median satisfies

$$
\widehat{\widehat{M e}\left[\widetilde{\mathbf{x}}_{\mathbf{n}}\right]}=\arg \min _{\widetilde{U} \in \mathcal{F}_{c}^{*}(\mathbb{R})} \frac{1}{n} \sum_{i=1}^{n}\left(\boldsymbol{\rho}_{\mathbf{1}}\left(\widetilde{x}_{i}, \widetilde{U}\right)\right),
$$

where for $\widetilde{U}, \widetilde{V}$

$$
\begin{gathered}
\rho_{\mathbf{1}}(\widetilde{U}, \widetilde{V})=\frac{1}{2} \int_{[0,1]}\left|\inf \widetilde{U}_{\alpha}-\inf \widetilde{V}_{\alpha}\right| d \alpha \\
+\frac{1}{2} \int_{[0,1]}\left|\sup \widetilde{U}_{\alpha}-\sup \widetilde{V}_{\alpha}\right| d \alpha
\end{gathered}
$$

(see Diamond and Kloeden [17]). 
In other words, the 1-norm median corresponds to an Mestimate associated with the loss function $\rho(x)=|x|$ and the $L^{1}$ distance $\rho_{\mathbf{1}}$.

Similarly, Sinova et al. [7] have introduced the $\varphi$ wabl/ldev/rdev median as follows.

Definition III.3. Let $\widetilde{\mathbf{x}}_{\mathbf{n}}=\left(\widetilde{x}_{1}, \ldots, \widetilde{x}_{n}\right)$ be a sample of independent observations from a random fuzzy number $\mathcal{X}$ : $\Omega \rightarrow \mathcal{F}_{c}^{*}(\mathbb{R})$ on a probability space $(\Omega, \mathcal{A}, P)$. Then the sample $\varphi$-wabl/ldev/rdev median of $\mathcal{X}$ is defined as the fuzzy number $\widetilde{\overline{\mathrm{Me}}}\left[\widetilde{\mathbf{x}}_{\mathbf{n}}\right] \in \mathcal{F}_{c}^{*}(\mathbb{R})$ such that for each $\alpha \in[0,1]$

$$
\begin{aligned}
& \left(\widetilde{\tilde{M}^{\varphi}\left[\widetilde{\mathbf{x}}_{\mathbf{n}}\right]}\right)_{\alpha}=\left[\operatorname{Me}\left\{\operatorname{wabl}^{\varphi}\left(\widetilde{x}_{1}\right), \ldots, \operatorname{wabl}^{\varphi}\left(\widetilde{x}_{n}\right)\right\}\right. \\
& -\operatorname{Me}\left\{\operatorname{ldev}_{\widetilde{x}_{1}}^{\varphi}(\alpha), \ldots, \operatorname{ldev}_{\widetilde{x}_{n}}^{\varphi}(\alpha)\right\}, \\
& \operatorname{Me}\left\{\operatorname{wabl}^{\varphi}\left(\widetilde{x}_{1}\right), \ldots, \operatorname{wabl}^{\varphi}\left(\widetilde{x}_{n}\right)\right\} \\
& \left.+\operatorname{Me}\left\{\operatorname{rdev}_{\widetilde{x}_{1}}^{\varphi}(\alpha), \ldots, \operatorname{rdev}_{\widetilde{x}_{n}}^{\varphi}(\alpha)\right\}\right]
\end{aligned}
$$

(where in case the real-valued median Me is non-unique the mid-point convention is considered).

The $\varphi$-wabl/ldev/rdev median satisfies

$$
\widehat{\widetilde{M} \varphi\left[\widetilde{\mathbf{x}}_{\mathbf{n}}\right]}=\arg \min _{\widetilde{U} \in \mathcal{F}_{c}^{*}(\mathbb{R})} \frac{1}{n} \sum_{i=1}^{n}\left(\mathscr{D}_{1}^{\varphi}\left(\widetilde{x}_{i}, \widetilde{U}\right)\right),
$$

where for $\widetilde{U}, \widetilde{V}$

$$
\begin{aligned}
& \mathscr{D}_{1}^{\varphi}(\widetilde{U}, \widetilde{V})=\left|\operatorname{wabl}^{\varphi}(\widetilde{U})-\operatorname{wabl}^{\varphi}(\widetilde{V})\right| \\
& +\frac{1}{2} \int_{[0,1]}\left|\operatorname{ldev}_{\widetilde{U}}^{\varphi}(\alpha)-\operatorname{ldev}_{\widetilde{V}}^{\varphi}(\alpha)\right| d \varphi(\alpha) \\
& +\frac{1}{2} \int_{[0,1]}\left|\operatorname{rdev}_{\widetilde{U}}^{\varphi}(\alpha)-\operatorname{rdev}_{\widetilde{V}}^{\varphi}(\alpha)\right| d \varphi(\alpha),
\end{aligned}
$$

with $\operatorname{wabl}^{\varphi}(\widetilde{U})=\int_{[0,1]} \operatorname{mid} \widetilde{U}_{\alpha} d \varphi(\alpha), \operatorname{ldev}_{\widetilde{U}}^{\varphi}(\alpha)=\operatorname{wabl}^{\varphi}(\widetilde{U})$ $-\inf \widetilde{U}_{\alpha}, \operatorname{rdev}_{\widetilde{U}}^{\varphi}(\alpha)=\sup \widetilde{U}_{\alpha}-\operatorname{wabl}^{\varphi}(\widetilde{U})$.

In other words, the $\varphi$-wabl/ldev/rdev median corresponds to an M-estimate associated with the loss function $\rho(x)=|x|$ and the $L^{1}$ distance $\mathscr{D}_{1}^{\varphi}$.

Since these ad hoc M-estimates have similar properties as the M-estimates obtained through the representer theorem, we make an empirical comparison in Section IV to investigate finite-sample differences.

\section{An illustrative real-life application}

We now consider a real-life example with fuzzy-valued data and compute the location M-estimates in Subsections III-A and III-C.

Example III.2. In 2011, TIMSS (Trends in International Mathematics and Science Study) and PIRLS (Progress in International Reading Literacy Study) assessments have joined for the first time to assess the fourth grade students in three fundamental curricular areas: mathematics, science, and reading.

The questionnaires in these studies are in a standard format, to be answered in accordance with a 4-point Likert scale, namely DISAGREE A LOT, DISAGREE A LITTLE, AGREE A LiTTLE and AGREE A LOT. As indicated in De la Rosa de
Sáa et al. [8], only limited conclusions can be obtained when the Likert scale responses are encoded by consecutive integer numbers.

On the other hand, the fuzzy rating scale has been introduced (Hesketh et al. [37]) as an approach that allows to combine a free-response format with a fuzzy valuation. In the fuzzy rating scale, along a continuous line between two endpoints

- a respondent first selects or draws a 'representative position/interval' of the respondent rating (i.e., the set of points which she/he considers to be fully compatible with such a rating),

- the respondent then also indicates 'latitudes of acceptance' on either side by determining the highest and lowest possible positions for the respondent rating (i.e., the set of points which she/he considers to be compatible to some extent with such a rating).

The fuzzy rating scale allows to explore and exploit more information than the Likert-scale. Moreover, from a statistical perspective the fuzzy rating scale is usually also more informative than the usual fuzzy linguistic approach (see e.g. Herrera et al. [38], Dong et al. [39], Rodríguez et al. [40] for some recent contributions to this approach), as shown by De la Rosa de Sáa et al. [8].

It can be argued that fuzzy rating scale-based questionnaires are not as user-friendly as Likert scales. However, minor training is usually sufficient to explain non-experts how to respond on a fuzzy rating scale (see Hesketh et al. [37]). To show how this fuzzy rating scale works by means of a simple example, some of the items for the Student questionnaire TIMSS/PIRLS (see http://timss.bc.edu/ timss2011/downloads/T11_StuQ_4.pdf) have been adapted in accordance with this scale, and the questionnaire has been conducted on the fourth grade students of the Colegio San Ignacio in Oviedo-Asturias (Spain). Note that the questions have not been modified with respect to the original questionnaire, but only the fuzzy rating scale has been added as a second way to respond. The students received instructions (in Spanish) on how to answer the questions (see http://carleos.epv.uniovi.es:8080/). We remark that this example accurately follows the spirit of Zadeh, who has coined it as the "precisiation of the imprecise" (see Zadeh [41]). Surprisingly, no answer has been slightly more frequent with the Likert than with the fuzzy rating scale.

We now analyze data from one of the considered items (a different and more detailed analysis of datasets from the whole adapted questionnaire can be found in Gil et al. [42]), referring to the degree of agreement with the statement that studying mathematics is harder than any other subject. The paper-andpencil format corresponding to this question is graphically displayed in Figure 4.

Data from the 68 fourth grade students from Colegio San Ignacio have been collected. The results are shown in the plot on the left in Figure 5. The outputs for the four location Mestimates discussed in the previous section are displayed in the plot on the right in Figure 5. 


\section{Mathematics in school}

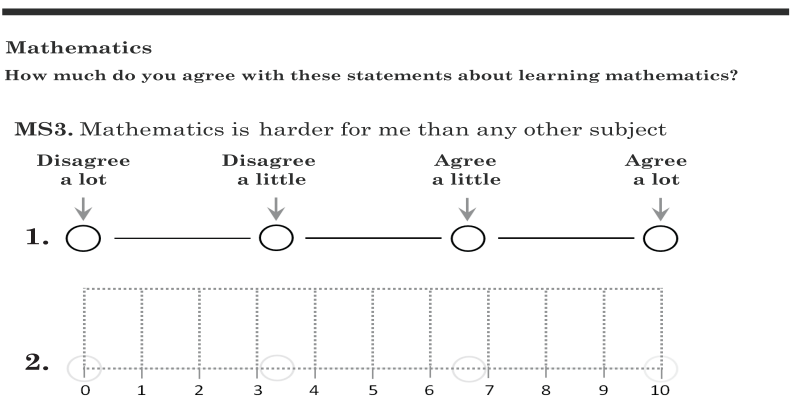

Fig. 4. An item about mathematics to fill out in the double-response questionnaire
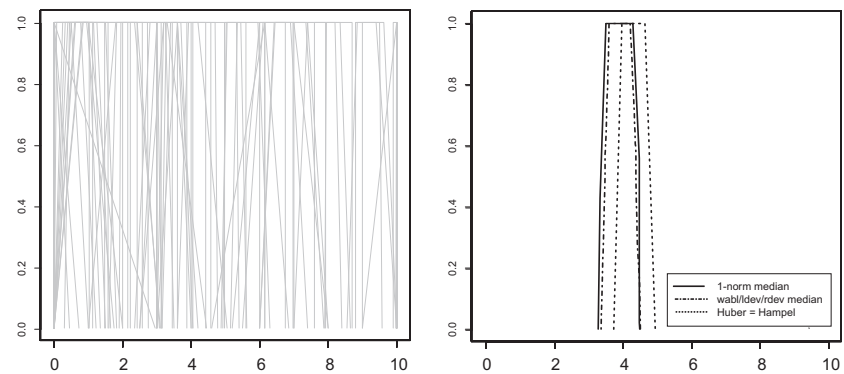

Fig. 5. Sample fuzzy data and location M-estimates of the 68 fuzzy rating scale-based responses to Question MS3

It can be seen that Hampel and Huber M-estimates, which are based on the $D_{1 / 3}^{\ell}$ metric and use the 1-norm median as initial solution, coincide.

It can be easily checked that the conditions for the representer theorem are satisfied for the Huber and Hampel Mestimates in this example, whence (as shown in Proposition III-B.3) they preserve the trapezoidal shape of the sample data.

This appealing property does not hold for the two mediantype M-estimates, as can be seen from Figure 5.

On the other hand, differences in the behavior of the $L^{1}$ type medians and the M-estimates in Figure 5 are mainly due to the influence of the corresponding loss functions. In this way, Huber and Hampel loss functions 'penalize' less (more) the small (large) distances than the absolute value.

It is interesting to comment that after integer encoding of the Likert-type responses, the medians for item MS3 correspond to AGREE A LITTLE (6. $\widehat{6})$. Hence, as already pointed out by De la Rosa de Sáa et al. [8], the fuzzy rating scale responses offer richer nuances and expressiveness.

In the literature one can find several arguments to employ triangular or trapezoidal fuzzy numbers or approximations preserving exactly or approximately ambiguity, expected interval, etc. (see, for instance, Pedrycz [44], Grzegorzewski [45], [46], [47], Grzegorzewski and Pasternak-Winiarska [48], Ban et al. [49], Lubiano et al. [50], and others). However, we emphasize that to compute the location M-estimation of fuzzy responses by means of the approaches in this paper, there is no need for fuzzy data to be trapezoidal/triangular (see e.g. Kosiński et al. [43] for some examples of other fuzzy data).

\section{EMPIRICAL COMPARATIVE ANALYSIS OF M-ESTIMATES WITH FUZZY DATA}

While it has been shown that the Aumann-type mean is not robust (Sinova et al. [6], [7]), the M-estimators do show robust behavior in terms of fsbp as discussed in Section III. In this section we empirically compare the performance and robustness of the fuzzy-valued M-estimators in more detail. In particular, we consider the M-estimators based on the Huber and Hampel loss functions using the $D_{1 / 3}^{\ell}$ and $D_{1}^{\ell}$ distances, as well as the 1-norm and $\ell$-wabl/ldev/rdev medians. For each of the estimators, the mean squared error of the estimator is approximated by simulation in different settings.

The general scheme of the simulations is as follows:

STUDY 1 (mid-points of the 1-levels generated from a symmetric distribution)

Based on four real-valued random variables, trapezoidal fuzzy data are generated according to $\mathcal{X}=\operatorname{Tra}\left(X_{1}-X_{2}\right.$ - $\left.X_{3}, X_{1}-X_{2}, X_{1}+X_{2}, X_{1}+X_{2}+X_{4}\right)$. Each sample contains a fraction $c_{p}$ of contaminated observations, where $c_{p}=0,0.1,0.2$, or 0.4 .

In the first case (CASE 1) the variables $X_{i}$ are independent. In particular,

- $X_{1} \sim \mathcal{N}(0,1)$ and $X_{2}, X_{3}, X_{4} \sim \chi_{1}^{2}$ for the regular observations.

- $X_{1} \sim \mathcal{N}(0,3)+C_{D}$ and $X_{2}, X_{3}, X_{4} \sim \chi_{4}^{2}+C_{D}$ for the contaminated observations.

In the second case (CASE 2) dependence between the variables $X_{i}$ is introduced as follows.

- $X_{1} \sim \mathcal{N}(0,1)$ and $X_{2}, X_{3}, X_{4} \sim 1 /\left(X_{1}^{2}+1\right)^{2}+0.1 \cdot \chi_{1}^{2}$ for the non-contaminated subsample (with $\chi_{1}^{2}$ independent of $X_{1}$ ),

- $X_{1} \sim \mathcal{N}(0,3)+C_{D}$ and $X_{2}, X_{3}, X_{4} \sim 1 /\left(X_{1}^{2}+1\right)^{2}$ $+0.1 \cdot \chi_{1}^{2}+C_{D}$ for the contaminated subsample (with $\chi_{1}^{2}$ independent of $X_{1}$ ).

The constant $C_{D}$, which determines the distance between the distribution of the regular and contaminated observations, equals $0,1,5,10$, or 100 . This yields 16 different settings for each case.

STUDY 2 (mid-points of the 1-levels generated from an asymmetric distribution)

For CASE 3, the trapezoidal fuzzy data are generated according to $\mathcal{X}=\operatorname{Tra}\left(X_{(1)}, X_{(2)}, X_{(3)}, X_{(4)}\right)$, with $X_{(1)}$ $\leq X_{(2)} \leq X_{(3)} \leq X_{(4)}$ the order statistics of $X_{1}, X_{2}, X_{3}, X_{4}$, which are distributed as

- $X_{1}, X_{2}, X_{3}, X_{4} \sim \operatorname{Beta}(5,1)$ for the regular observations.

- $X_{1}, X_{2}, X_{3}, X_{4} \sim \operatorname{Beta}\left(1, C_{D}+1\right)$ for the contaminated observations.

Trapezoidal fuzzy data are generated as in STUDY 1, but now $X_{i}$ are distributed as

- $X_{1} \sim \operatorname{Beta}(5,1), X_{2} \sim$ Uniform $\left[0, \min \left\{X_{1}, 1-X_{1}\right\}\right]$, $X_{3} \sim$ Uniform $\left[0, X_{1}-X_{2}\right]$ and $X_{4} \sim$ Uniform $[0,1$ $\left.-X_{1}-X_{2}\right]$ for the regular observations. 
TABLE I

M-ESTIMATE SHOWING THE LOWEST ESTIMATED MEAN SQUARED ERROR FOR THE SIMULATION DESIGNS IN STUDIES 1 AND 2

\begin{tabular}{|c|c|c|c|c|c|c|c|c|c|c|c|c|c|c|c|c|c|}
\hline \multirow[b]{2}{*}{$c_{p}$} & \multirow[b]{2}{*}{$C_{D}$} & \multicolumn{4}{|c|}{ STUDY 1 - CASE 1} & \multicolumn{4}{|c|}{ STUDY 1 - CASE 2} & \multicolumn{4}{|c|}{ STUDY 2 - CASE 3} & \multicolumn{4}{|c|}{ STUDY 2 - CASE 4} \\
\hline & & $\rho_{1}$ & $\mathscr{D}_{1}^{\ell}$ & $D_{1 / 3}^{\ell}$ & $\rho_{2}$ & $\rho_{1}$ & $\mathscr{D}_{1}^{\ell}$ & $D_{1 / 3}^{\ell}$ & $\rho_{2}$ & $\rho_{1}$ & $\mathscr{D}_{1}^{\ell}$ & $D_{1 / 3}^{\ell}$ & $\rho_{2}$ & $\rho_{1}$ & $\mathscr{D}_{1}^{\ell}$ & $D_{1 / 3}^{\ell}$ & $\rho_{2}$ \\
\hline 0.1 & 0 & HAMP ${ }^{*}$ & HAMP* & HAMP* & HAMP* & $1-n$ & 1-n & $1-n$ & $1-n$ & HAMP* & HAMP* & HAMP* & HAMP* & HAMP & HAMP & HAMP & HAMP \\
\hline 0.1 & 1 & HAMP & HAMP & HAMP & HAMP & $1-n$ & $1-n$ & HAMP* & $1-n$ & HAMP & HAMP & HAMP & HAMP & HAMP & HAMP & HAMP & HAMP \\
\hline 0.1 & 5 & HAMP & HAMP & HAMP & HAMP & $1-n$ & $1-n$ & 1-n & $1-n$ & HAMP & HAMP & HAMP & HAMP & HAMP & HAMP & HAMP & HAMP \\
\hline 0.1 & 10 & HAMP & HAMP & HAMP & HAMP & $1-n$ & $1-n$ & $1-n$ & $1-n$ & HAMP & HAMP & HAMP & HAMP & HAMP & $1-n$ & $1-n$ & HAMP \\
\hline 0.1 & 100 & HAMP & HAMP & HAMP & HAMP & $1-n$ & $1-n$ & HAMP & $1-n$ & HAMP & HAMP & HAMP & HAMP & HAMP* & HAMP* & HAMP $^{*}$ & HAMP $^{*}$ \\
\hline 0.2 & 0 & HAMP $^{*}$ & HAMP* & HAMP* & HAMP* & $1-n$ & $1-n$ & $1-n$ & $1-n$ & HAMP & HAMP & HAMP & HAMP & HAMP & HAMP* & HAMP* & HAMP* \\
\hline 0.2 & 1 & HAMP* & HAMP* & HAMP* & HAMP* & $1-n$ & HAMP* & HAMP* & HAMP* & HAMP & HAMP & HAMP & HAMP & HAMP & 1-n & 1-n & HAMP \\
\hline 0.2 & 5 & HAMP & HAMP & HAMP & HAMP & $1-n$ & 1-n & $1-n$ & 1-n & HAMP & HAMP & HAMP & HAMP & HAMP & HAMP & HAMP & HAMP \\
\hline 0.2 & 10 & HAMP & HAMP & HAMP & HAMP & $1-n$ & $1-n$ & $1-n$ & $1-n$ & HAMP & HAMP & HAMP & HAMP & HAMP* & HAMP & HAMP ${ }^{*}$ & HAMP \\
\hline 0.2 & 100 & HAMP & HAMP & HAMP & HAMP & $1-n$ & $1-n$ & $1-n$ & $1-n$ & HAMP & HAMP & HAMP & HAMP & HAMP $^{*}$ & HAMP* & HAMP $^{*}$ & HAMP* \\
\hline 0.4 & 0 & HAMP ${ }^{*}$ & $\mathrm{w} / \mathrm{l} / \mathrm{r}$ & $\mathrm{w} / \mathrm{l} / \mathrm{r}$ & HAMP* & $1-n$ & $1-n$ & $1-n$ & $1-n$ & HAMP & HAMP & $1-n$ & HAMP & HAMP ${ }^{*}$ & HAMP* & HAMP* & HAMP $^{*}$ \\
\hline 0.4 & 1 & HAMP* & HAMP* & HAMP* & HAMP* & $1-n$ & $1-n$ & $1-n$ & $1-n$ & HAMP & HAMP & HAMP & HAMP & HAMP* & HAMP* & HAMP ${ }^{*}$ & HAMP \\
\hline 0.4 & 5 & HAMP ${ }^{*}$ & $\mathrm{w} / \mathrm{l} / \mathrm{r}$ & $\mathrm{w} / \mathrm{l} / \mathrm{r}$ & $\mathrm{w} / \mathrm{l} / \mathrm{r}$ & $1-n$ & $1-n$ & $1-n$ & $1-n$ & HAMP & HAMP & HAMP & HAMP & HAMP & $\mathrm{w} / \mathrm{l} / \mathrm{r}$ & $\mathrm{w} / \mathrm{l} / \mathrm{r}$ & HAMP \\
\hline 0.4 & 10 & HAMP* & HAMP* & HAMP* & $\mathrm{w} / \mathrm{l} / \mathrm{r}$ & $1-n$ & $1-n$ & $1-n$ & $1-n$ & HAMP & HAMP & HAMP & HAMP & $\mathrm{w} / \mathrm{l} / \mathrm{r}$ & $\mathrm{w} / \mathrm{l} / \mathrm{r}$ & $\mathrm{w} / \mathrm{l} / \mathrm{r}$ & HUB \\
\hline 0.4 & 100 & HAMP $^{*}$ & HAMP* & $\mathrm{w} / \mathrm{l} / \mathrm{r}$ & $\mathrm{w} / \mathrm{l} / \mathrm{r}$ & $1-n$ & $1-n$ & $1-n$ & $1-n$ & HAMP & $1-n$ & $1-n$ & HAMP & $\mathrm{w} / \mathrm{l} / \mathrm{r}$ & $\mathrm{w} / \mathrm{l} / \mathrm{r}$ & $\mathrm{w} / \mathrm{l} / \mathrm{r}$ & HUB \\
\hline
\end{tabular}

- $X_{1} \sim \operatorname{Beta}\left(1, C_{D}+1\right), X_{2} \sim \min \left\{X_{1}, 1-X_{1}\right\}$. $\operatorname{Beta}\left(1, C_{D}+1\right), X_{3} \sim\left(X_{1}-X_{2}\right) \cdot \operatorname{Beta}\left(1, C_{D}+1\right)$ and $X_{4} \sim\left(1-X_{1}-X_{2}\right) \cdot \operatorname{Beta}\left(1, C_{D}+1\right)$ for the contaminated observations.

The same values of $c_{p}$ and $C_{D}$ as in STUDY 1 are considered.

For each of the four cases above, the simulation study was performed according to the following steps.

Step 1. To determine the population analog of the M-estimates for each case, $N=1000$ samples of size $n=10000$, consisting of only regular fuzzy-number observations $\left(c_{p}=C_{D}=0\right)$ have been generated. The population target for each M-estimator is then taken as the Monte Carlo average of the corresponding M-estimates in these $N$ samples.

Step 2. For each case and each combination of $c_{p}$ and $C_{D}$, we then generated $N=1000$ random samples and calculated the corresponding M-estimates. Based on these M-estimates, the mean squared error w.r.t. the population targets has been computed for each location measure, using four different metrics, namely $\rho_{1}, \mathscr{D}_{1}^{\ell}, D_{1 / 3}^{\ell}$, and $\rho_{2}$.

The results of our comparative analysis are summarized in Table I, which lists for each setting the $\mathrm{M}$ estimator that yielded the lowest estimated mean squared error. We used the abbreviations HAMP = M-estimator with Hampel loss function, $\mathrm{HUB}=\mathrm{M}$-estimator with $\mathrm{Hu}$ ber loss function, $1-\mathrm{n}=1$-norm median, $\mathrm{w} / \mathrm{l} / \mathrm{r}=\boldsymbol{\varphi}$ wabl/ldev/rdev median. The notation $*$ distinguishes the Hampel and Huber M-estimators computed by means of the $D_{1}^{\ell}$ distance. The detailed results from the simulations can be found at http://bellman.ciencias.uniovi.es/SMIRE/ Archivos/SimulationsMestimates.pdf.

From Table I it becomes clear that there does not exist a uniformly most appropriate M-estimator of location. However, the M-estimator based on Hampel's loss function seems to be the most suitable estimator in most of the situations when the trapezoidal fuzzy data are assumed to be generated from independent real random variables. In the case that these variables are dependent, the 1-norm median seems to be most successful when mid-points are generated from a symmetric distribution, whereas there is a high diversity for asymmetrically distributed mid-points.

Remark IV.1. In addition to the empirical advantages of the M-estimators based on Hampel's loss function, it should be highlighted that thanks to the representer theorem, the Mestimates of important classes of fuzzy data such as trapezoidal data are guaranteed to give a location estimate that belongs to the same class. This is because the estimates are expressible as weighted averages. This result is not generally true for $a d$ hoc robust measures, which complicates their interpretation as measure of the location of the fuzzy data.

\section{CONCLUDing REMARKS}

One of the advantages of the new location M-estimators for random fuzzy numbers introduced in this paper lies in the fact that the recently established representer theorem in the context of robust density estimation could be easily extended to the case of fuzzy -number data, as shown in Section III. Although the M-estimates based on the representer theorem have been developed for the $D_{\theta}^{\varphi}$ metric, other $L^{2}$ metrics between fuzzy numbers allowing to embed $\mathcal{F}_{c}^{*}(\mathbb{R})$ into a convex cone of a Hilbert space are also worthwhile to consider. For instance, in most of the examples and simulations in this paper we have also considered the $L^{2}$ metric based on the wabl/ldev/rdev representation introduced in Sinova et al. [3], but the conclusions were essentially the same.

It should be pointed out that, in spite of the fact that the mathematical aparatus behind the M-estimates in Subsections III-A and III-B involves the Hilbert space-valued setting, the particular case of fuzzy data is definitely much simpler and easier-to-follow. Furthermore, due to the representer theorem, the computations reduce to the calculus of weighted means. The main practical difficulty concerns the calculus of the involved weights, but this iterative procedure can be implemented in R [55] or similar tools.

Finally, an important remaining problem is to adapt the Mestimates of location with unknown dispersion to the fuzzyvalued case. This would formally overcome the lack of scale equivariance for the location M-estimators. Therefore, the tuning constant(s) in the loss functions would not need to be 
determined from the data anymore. We hope to solve this issue in the future, when robust dispersion measures for fuzzy data are also developed.

\section{REFERENCES}

[1] M.L. Puri and D.A. Ralescu, "Fuzzy random variables," J. Math. Anal. Appl., Vol. 114, pp. 409-422, 1986.

[2] G. González-Rodríguez, A. Colubi and M.A. Gil, "Fuzzy data treated as functional data: A one-way ANOVA test approach," Comput. Stat. Data Anal. Vol. 56, no. 4, pp. 943-955, 2012.

[3] B. Sinova, M.A. Gil, M.T. López and S. Van Aelst, "A parameterized $L^{2}$ metric between fuzzy numbers and its parameter interpretation," Fuzzy Sets Syst., Vol. 245, pp. 101-115, 2014.

[4] J.S. Kim and C.D. Scott, "On the robustness of kernel density Mestimators," in: Proc. 28th Int. Conf. Mach. Lear. Bellevue, Washington, 2011, pp. 697-704.

[5] J.S. Kim and C.D. Scott, "Robust kernel density estimation," J. Mach. Learn. Res., Vol. 13, 2012, pp. 2529-2565.

[6] B. Sinova, M.A. Gil, A. Colubi and S. Van Aelst, "The median of a random fuzzy number. The 1-norm distance approach," Fuzzy Sets Syst., Vol. 200, pp. 99-115, 2012.

[7] B. Sinova, S. de la Rosa de Sáa and M.A. Gil, "A generalized $L^{1}$-type metric between fuzzy numbers for an approach to central tendency of fuzzy data," Inf. Sci., Vol. 242, pp. 22-34, 2013.

[8] S. De la Rosa de Sáa S, M.A. Gil, G. González-Rodríguez, M.T. López and M.A. Lubiano, "Fuzzy rating scale-based questionnaires and their statistical analysis," IEEE Trans. Fuzzy Syst., Vol. 23, no. 1, pp. 111126, Feb. 2015

[9] C. Bertoluzza, N. Corral and A. Salas, "On a new class of distances between fuzzy numbers," Math \& Soft Comput., Vol. 2, pp. 71-84, 1995.

[10] M.R. Casals, N. Corral, M.A. Gil, M.T. López, M.A. Lubiano, M. Montenegro, G. Naval and A. Salas, "Bertoluzza et al.'s metric as a basis for analyzing fuzzy data," Metron, Vol. 71, no. 3, pp. 307-322, 2013.

[11] W. Trutschnig, G. González-Rodríguez, A. Colubi and M.A. Gil, "A new family of metrics for compact, convex (fuzzy) sets based on a generalized concept of mid and spread," Inf. Sci., Vol. 179, pp. 3964-3972, 2009.

[12] A. Blanco-Fernández, M.R. Casals, A. Colubi, N. Corral, M. GarcíaBárzana; M.A. Gil, G. González-Rodríguez, M.T. López, M. Montenegro, M.A. Lubiano, A.B. Ramos-Guajardo, S. de la Rosa de Sáa and B. Sinova, "Random fuzzy sets: a mathematical tool to develop statistical fuzzy data analysis," Iran. J. Fuzzy Syst., Vol. 10, no. 2, pp. 1-28, 2013.

[13] A. Blanco-Fernández, M.R. Casals, A. Colubi, R. Coppi, N. Corral, S. de la Rosa de Sáa, P. D’Urso, M.B. Ferraro, M. García-Bárzana, M.A. Gil, P. Giordani, G. González-Rodríguez, M.T. López, M.A. Lubiano, M. Montenegro, T. Nakama, A.B. Ramos-Guajardo, B. Sinova and W. Trutschnig, "Arithmetic and distance-based approach to the statistical analysis of imprecisely valued data," in: Towards Advanced Data Analysis by Combining Soft Computing and Statistics. Stud. Fuz. Soft Comp. 285. Heidelberg: Springer, 2013, pp. 1-18

[14] A. Blanco-Fernández, M.R. Casals, A. Colubi, N. Corral, M. GarcíaBárzana, M.A. Gil, G. González-Rodríguez, M.T. López, M.A. Lubiano, M. Montenegro, A.B. Ramos-Guajardo, S. de la Rosa de Sáa and B. Sinova, "A distance-based statistical analysis of fuzzy number-valued data," Int. J. Approx. Reas., Vol. 55, pp. 1487-1501, 2014.

[15] A. Blanco-Fernández, M.R. Casals, A. Colubi, N. Corral, M. GarcíaBárzana, M.A. Gil, G. González-Rodríguez, M.T. López, M.A. Lubiano, M. Montenegro, A.B. Ramos-Guajardo, S. de la Rosa de Sáa and B. Sinova, "Rejoinder on "A distance-based statistical analysis of fuzzy number-valued data"'. Int. J. Approx. Reas., Vol. 55, pp. 1601-1605, 2014.

[16] M.A. Gil, A. Colubi and P. Terán, "Random fuzzy sets: why, when, how," BEIO, Vol. 30, no. 1, 2013, pp. 5-29.

[17] P. Diamond and P. Kloeden, "Metric spaces of fuzzy sets," Fuzzy Sets Syst., Vol. 35, 1990, pp. 241-249.

[18] M.L. Puri and D.A. Ralescu, "The concept of normality for fuzzy random variables," Ann. Probab., Vol. 11, pp. 1373-1379, 1985.

[19] M. Fréchet, "Les éléments aléatoires de nature quelconque dans un espace distancié," Ann. L'Inst. H. Poincaré, Vol. 10, pp. 215-310, 1948.

[20] R. Féron, "Ensembles aléatoires flous," C.R. Acad. Sc. Paris A, Vol. 282, 1976, pp. 903-906.

[21] A. Colubi, M. López-Díaz, M., J.S. Domínguez-Menchero and M.A. Gil, "A generalized Strong Law of Large Numbers," Probab. Theory Relat. Fields, Vol. 114, pp. 401-417, 1999.

[22] P.J. Huber, "Robust estimation of a location parameter," Ann. Math. Statist., Vo. 35, no. 1, 1964, pp. 73-101.
[23] P.J. Huber, Robust Statistics. New York: J. Wiley \& Sons, 1981.

[24] D.G. Luenberger, Optimization by Vector Space Methods. New York: Wiley-Interscience, 1997.

[25] S. Wang, J. Zhu, F.L. Chung, Q. Lin and D. Hu, "Theoretically optimal parameter choices for support vector regression machines with noisy input," Soft Comput., Vol. 9, 2005, pp. 732-741.

[26] M. Debruyne, M. Hubert and J.A.K. Suykens, "Model selection in kernel based regression using the influence function," J. Mach. Learn. Res., Vol. 9, 2008, pp. 2377-2400.

[27] F.R. Hampel, "The influence curve and its role in robust estimation," $J$. Amer. Stat. Assoc. Vol. 69, no. 346, 1974, pp. 383-393.

[28] R.A. Maronna, R.D. Martin and V.J. Yohai, Robust Statistics: Theory and Methods. Chinchester: J. Wiley \& Sons, 2006.

[29] D. Dubois and H. Prade, "Additions of interactive fuzzy numbers," IEEE Trans. Automat. Control, Vol. 26, pp. 926-936, Aug. 1981.

[30] B. Sinova, M.R. Casals and M.A. Gil, "Central tendency for symmetric random fuzzy numbers," Inform. Sci., Vol. 278, pp. 599-613, 2014.

[31] R.A. Vandermeulen and C.D. Scott, "Consistency of robust kernel density estimators," JMLR: Works. and Conf. Proc., Vol. 30, pp. 1-24, 2013.

[32] F.R. Hampel, " A general qualitative definition of robustness," Ann. Math. Stat., Vol. 42, no. 6, 1971, pp. 1887-1896.

[33] D.L. Donoho and P.J. Huber, "The notion of breakdown point," in: $A$ Festschrift for Erich L. Lehmann (P.J. Bickel, K. Doksum and J.L. Hodges Jr, Eds). Belmont: Wadsworth, 1983, pp. 157-184

[34] A. Cuevas, M. Febrero and R. Fraiman, "Robust estimation and classification for functional data via projection-based depth notions," Comput. Statist., Vol. 22, 2007, pp. 481-496.

[35] P.J. Huber, "Finite Sample Breakdown of M- and P-Estimators," Ann. Statist., Vol. 12, no. 1, 1984, pp. 119-126.

[36] N. Corral-Blanco, E. Zurbano-Fernández, A. Blanco-Fernández, I. García-Honrado and A.B. Ramos-Guajardo, "Structure of the family educational environment: its influence on performance and differential performance," in: PIRLS - TIMSS 2011 International Study on Progress in Reading Comprehension, Mathematics and Sciences IEA. Volume II. Spanish Report. Secondary Analysis. Madrid: Ministerio de Educación, Cultura y Deporte, Instituto Nacional de Evaluación Educativa, 2013, pp. 9-31 (http://www.mecd.gob.es/dctm/inee/internacional/pirlstimss2011eng1.pdf?documentld=0901e72b81825be3).

[37] T. Hesketh, R. Pryor and B. Hesketh, "An application of a computerized fuzzy graphic rating scale to the psychological measurement of individual differences," Int. J. Man-Mach. Stud., Vol. 29, 1988, pp. 21-35.

[38] F. Herrera, E. Herrera-Viedma and L. Martínez, "A fuzzy linguistic methodology to deal with unbalanced linguistic term sets," IEEE Trans. Fuzzy Syst., Vol. 16, no. 2, pp. 354-370, Apr. 2008.

[39] Y. Dong, W.-C. Hong, Y. Xu and S. Yu, "Selecting the individual numerical scale and prioritization method in the analytic hierarchy process: A 2-Tuple fuzzy linguistic approach," IEEE Trans. Fuzzy Syst., Vol. 19, no. 1, pp. 13-25, Feb. 2011

[40] R.M. Rodríguez, L. Martínez and F. Herrera, "Hesitant fuzzy linguistic term sets for decision making," IEEE Trans. Fuzzy Syst., Vol. 20, no. 1, pp. 109-119, Feb. 2012.

[41] L.A. Zadeh, "Is there a need for fuzzy logic?," Inform. Sci., Vol. 178, 2008, pp. 2751-2779.

[42] M.A. Gil, M.A. Lubiano, S. de la Rosa de Sáa and B. Sinova, "Analyzing data from a fuzzy rating scale-based questionnaire. A case study," Psicothema, Vol. 27, no. 2, pp. 182-191, 2015.

[43] W. Kosiński, P. Prokopowicz and A. Rosa, "Defuzzification functionals of ordered fuzzy numbers," IEEE Trans. Fuzzy Syst., Vol. 21, no. 6, pp. 1163-1169, Dec. 2013.

[44] W. Pedrycz, "Why triangular membership functions?," Fuzzy Sets Syst., Vol. 64, no. 1, pp. 21-30, 1994.

[45] P. Grzegorzewski, "Trapezoidal approximations of fuzzy numbers preserving the expected interval - algorithms and properties," Fuzzy Sets Syst., Vol. 159, pp. 1354-1364, 2008.

[46] P. Grzegorzewski, "Algorithms for trapezoidal approximations of fuzzy numbers preserving the expected interval," in Foundations of Reasoning under Uncertainty. Berlin: Springer, 2010, pp. 85-98.

[47] P. Grzegorzewski, "Fuzzy number approximation via shadowed sets," Inf. Sci., Vol. 225, pp. 35-46, 2013.

[48] P. Grzegorzewski and K. Pasternak-Winiarska, "Trapezoidal approximations of fuzzy numbers with restrictions on the support and core," in: Proc. 7th Conf. EUSFLAT-2011 and LFA-2011. Paris: Atlantis Press, 2011, pp. 749-756.

[49] A. Ban, L. Coroianu and P. Grzegorzewski, "Trapezoidal approximation and aggregation,” Fuzzy Sets Syst., Vol. 177, pp. 45-59, 2011. 
[50] M.A. Lubiano, S. De la Rosa de Sáa, B. Sinova and M.A. Gil, "Empirical sensitivity analysis on the influence of the shape of fuzzy data on the estimation of some statistical measures," in: Strengthening Links Between Data Analysis and Soft Computing. Adv. Intel. Syst. Comp. 315 (P. Grzegorzewski, M. Gagolewski, O. Hryniewicz and M.A. Gil, Eds). Heidelberg: Springer, 2015, pp. 123-131.

[51] J. Serrano-Guerrero, E. Herrera-Viedma, J.A. Olivas, A. Cerezo and F.P. Romero, "A google wave-based fuzzy recommender system to disseminate information in University Digital Libraries 2.0," Inform. Sci., Vol. 181, pp. 1503-1516, 2011.

[52] C. Porcel, A. Tejeda-Lorente, M.A. Martínez and E. Herrera-Viedma, "A hybrid recommender system for the selective dissemination of research resources in a Technology Transfer Office," Inform. Sci., Vol. 184, pp. $1-19,2012$

[53] J.R. Chou, "A linguistic evaluation approach for universal design,". Inform. Sci., Vol. 190, pp. 76-94, 2012.

[54] L.A. Zadeh, "Fuzzy logic = computing with words," IEEE Trans. Fuzzy Syst., Vol. 4, no. 2, pp. 103-111, 1996.

[55] R Core Team, R: A Language and Environment for Statistical Computing, R Foundation for Statistical Computing, Vienna, Austria, 2015 (http://www.r-project.org).

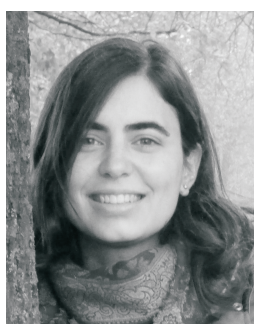

Beatriz Sinova received her BSc and MSc degrees in Mathematics in 2009 and 2010, respectively, from the University of Oviedo, Spain. In 2014, she has received a Joint $\mathrm{PhD}$ degree in Mathematics and Statistics from the Universities of Oviedo and Gent. Her current research interests include the development of new statistical techniques for the location of imprecise-valued data, as well as their application to real-life problems. She is involved in some research projects regarding the statistical analysis of interval-, fuzzy- and functional-valued data.

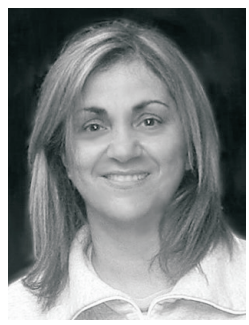

María Ángeles Gil is full professor of Statistics and O.R. at the University of Oviedo (Spain) since 1992. Her research was initially focused on the measurement and statistical developments concerning the amount of information and inequality of random variables. Since 1983, her research interests have been mostly related to the statistical analysis of imprecise data (mainly set-valued and fuzzy setvalued). On this topic she heads the research group SMIRE (http://bellman.ciencias.uniovi.es) and she is a member of the Core SMPS Group steering the biennial conferences on Soft Methods in Probability and Statistics. She was co-editor-in-chief of the journal TEST (2005-2008), and is currently associate/area editor for Computational Statistics and Data Analysis, Fuzzy Sets and Systems, Information Sciences, International Journal of Uncertainty, Fuzziness and Knowledge-Based Systems, International Transactions in Operational Research, and TEST. She has been awarded with the Silver Medal of the Principality of Asturias in 2014 and is an IFSA fellow since 2015.

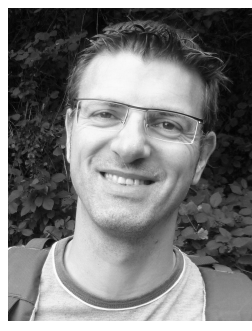

Stefan Van Aelst Stefan Van Aelst received the B.Sc. and M.Sc. degrees in mathematics from the University of Antwerp, Belgium, in 1997 and the $\mathrm{Ph} . \mathrm{D}$. degree (also in mathematics) from the University of Antwerp, Belgium, in 2000. He has been assistant and associate professor in statistics at Ghent University. Since 2013 he is associate professor in statistics in the department of Mathematics at the University of Leuven (KULeuven) and part-time associate professor at Ghent University his research is mainly focused on robust statistics, covering estimation, inference, bootstrap methods, model selection, the analysis of fuzzy data and high-dimensional data analysis. 